\title{
Review of: "Durability of SARS-CoV-2-specific T cell responses at 12-months post-infection"
}

\author{
Alejandro Vallejo
}

Potential competing interests: The author(s) declared that no potential competing interests exist.

This is a well written manuscript indicating protection 12 months post infection, with higher induction of polyfunctional memory T cells in those who experience severe disease especially in specific CD4 cells. The methods are well defined encompassing humoral and cell responses. The manuscript highlights that SARSCoV-2-specific CD4 T cells present in individuals infected 12-months prior exhibit differentiation toward central memory phenotypes, activation by expression of CD69 and PD-1, and polyfunctionality by expression of IFNg and IL2. This suggests that the breadth of T cell epitope recognition by these memory $T$ cells may provide durable protection against emerging SARS-CoV-2 variants. 